\title{
ENDOTHELIUM-INDEPENDENT DILATION IN CHILDREN AND ADOLESCENTS
}

\author{
A THESIS \\ SUBMITTED TO THE FACULTY OF THE GRADUATE SCHOOL \\ OF THE UNIVERSITY OF MINNESOTA \\ BY:
}

Kara Lynn Marlatt

\begin{abstract}
IN PARTIAL FULFILLMENT OF THE REQUIREMENTS FOR THE DEGREE OF:

Master of Science
\end{abstract}

ADVISOR: Donald R. Dengel, Ph.D.

August 2011 
Copyright Page

(c) Kara Lynn Marlatt 2011. All rights reserved. 


\section{ACKNOWLEDGEMENTS}

The following thesis was prepared under the direction of Donald R. Dengel,

Ph.D., Aaron S. Kelly, Ph.D., and George R. Biltz, M.D., at the University of Minnesota, as well as the assistance from Andrea M. Metzig, M.A., Julia Steinberger, M.S., M.D., and Meghan C. McCue, B.A.

I wish to thank each of them individually for their contributions to my work, as well as guiding me through the process of publishing a shortened version of this thesis as a manuscript within Clinical Physiology and Functional Imaging. Without their help, I would not have the knowledge and structural support to allow me to create such works as this at this stage in my career. Thank you. 


\section{ABSTRACT}

Peak brachial artery dilation post-nitroglycerin (NTG) administration occurs between 3 and 5 -min in adults. The purpose of this study was to identify the time to peak dilation response to sublingual NTG $(0.3 \mathrm{mg})$ in youth. Endotheliumindependent dilation (EID) was measured in 198 healthy (113 males, 85 females) youth (6-18 yrs) via ultrasound imaging of the brachial artery following NTG administration up to 5-min. Time to peak EID was 4-min, 28-sec, following NTG administration. There was a significant $(p<0.001)$ difference post-NTG at the 3 vs. 4 -min, 4 vs. 5 -min, and 3 vs. 5 -min time points. Peak EID (males: $24.8 \pm 0.5$ vs. females: $25.3 \pm 0.6 \%, p=0.6)$ was not significantly different between genders after adjusting for baseline brachial diameter. Endothelium-independent dilation was measured up to 8 -min in a small sub-group of 20 youth (12 males, 8 females), resulting in a time to peak dilation of 4-min, $24-\mathrm{sec}$, and an average change in peak dilation of $23.8 \pm 1.2 \%$. No significant difference existed beyond the 4-min time point within the small sub-group. In conclusion, peak response to NTG administration occurred between 4 and 5-min. The results demonstrate the importance of measuring EID up to 5-min post-nitroglycerin administration in youth.

Key words: Ultrasound, Nitroglycerin, Endothelium-Independent Dilation, EID, Smooth Muscle, Vascular Function. 
TABLE OF CONTENTS

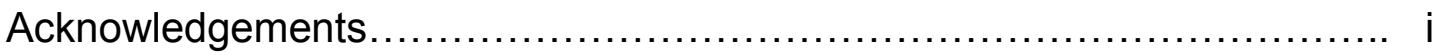

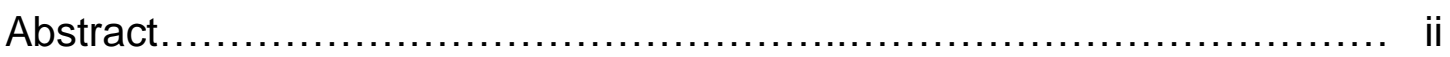

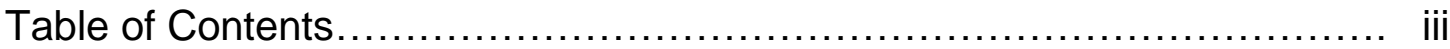

List of Tables................................................................... iv

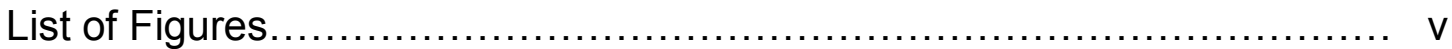

Chapter 1. Introduction................................................. 1

Chapter 2. Review of Literature ......................................... 5

Endothelium Response to Shear Stress............................ 7

Flow-Mediated, or Endothelium-Dependent Dilation................... 9

Endothelium-Independent Dilation by Nitroglycerin Administration..... 10

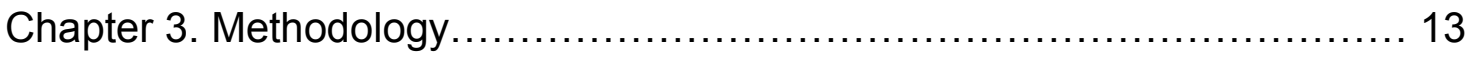

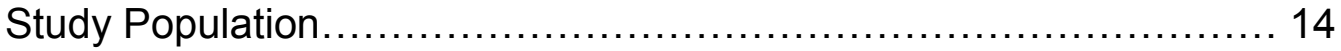

Measurements........................................................ 15

Statistical Analysis..................................................... 17

Chapter 4. Results........................................................ 19

Chapter 5. Discussion........................................................... 22

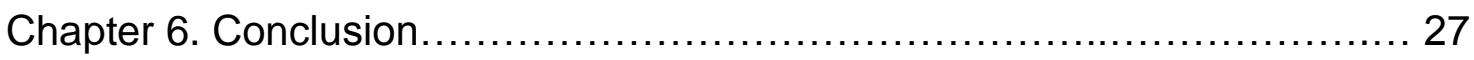

Chapter 7. References ........................................................ 29 


\section{LIST OF TABLES}

Table 1. Mean ( \pm Standard Error of the Mean) Demographic Characteristics..........41

Table 2. P-values Among Gender and Tanner Stage Stratifications..................42 


\section{LIST OF FIGURES}

Figure 1. Endothelium-Independent Dilation Time Course Over 5 Minutes..........43

Figure 2. Endothelium-Independent Dilation Time Course Over 8 Minutes..........44 
CHAPTER 1. INTRODUCTION 
Prior to the 1980s, the endothelium was thought only to be a physical barrier between the blood vessel wall and the blood stream. Research in the 1980 s and early 1990s revealed the endothelium's role as a much more dynamic facilitator of vascular relaxation, or dilation. Moreover, an increase in blood flow was discovered to facilitate a dilatory response within a normal, healthy blood vessel.

Brachial artery response to flow-mediated dilation (FMD) assessment with ultrasound, also known as endothelium-dependent dilation (EDD), became the standard means of non-invasively evaluating vascular response. Following forearm occlusion for several minutes, the endothelium's response to increased blood flow and subsequent vasodilator effects can be effectively analyzed. Similarly, endothelium-independent dilation (EID) assessment has also been considered a well-validated means of assessing smooth muscle sensitivity to a nitric oxide donor. Unlike EDD, EID utilizes nitroglycerin (NTG) to ensure that abnormalities of EDD are truly the result of endothelial dysfunction (i.e. poor dilation response as a result of reduced endogenously-produced nitric oxide). While research examining adult populations reported maximal brachial artery dilation response 3 to 5 -min post-NTG administration, EID response in children and adolescents has not been studied. Therefore, the purpose of the study was to describe the time course of brachial artery dilation response to a fixed dose of sublingual NTG, identify the time point at which peak dilation occurs in children 
and adolescents between the ages of 6 and 18 years, and assess any gender differences in this response. We hypothesized that the time course of EID would be similar in children as has been described in adults, and that the smaller resting brachial artery diameter in females would result in higher peak EID response compared to males.

A complete literature review, as well as a subsequent methodology, results, discussion, and conclusion surrounding the proposed study are detailed in the following chapters:

Chapter two summarizes the current literature related to EDD, documenting the technique as a well-validated means of assessing endothelial function. Healthy and disease population differences in EDD will be discussed. Additionally, EID will be introduced as a measure of the exogenous effect of NTG on smooth muscle relaxation, with the current literature clearly stated.

Chapter three addresses the methodology of this study. Background information surrounding the study population; data abstraction and measurement techniques, including anthropometric, blood pressure, and vascular assessments; as well as statistical analysis are stated. 
Chapter four details the findings of this study, by examining the time to peak dilation, maximal dilation, difference between dilation response to NTG at different time points along the time course, as well as possible age, race, and gender differences among the study population.

Chapter five summarizes and discusses the implications of these findings with reference to the current literature. Results of gender differences before and after baseline diameter adjustment, as well as age and Tanner "pubertal" stage adjustments, are discussed. Further discussion of gender differentiation is also reviewed.

Chapter 6 offers conclusions of the resulting study, as well as states the necessity for future research within EID assessment in children and adolescents. 
CHAPTER 2. REVIEW OF LITERATURE 
The endothelium is an organ that has both an autocrine and paracrine role in the control of vascular tone, smooth muscle proliferation, platelet aggregation, monocyte and leukocyte adhesion, and thrombosis (Anderson et al., 1995; Cines et al., 1998; Yoshida et al., 2006). Before the 1980s, endothelial cells lying within the intima layer of blood vessels were thought to merely act as a physical barrier between the vessel wall and the blood stream, regulating delivery of blood to and from tissues. However, a discovery in 1978 by Dr. Robert Furchgott helped characterize a critical substance in the endothelial cells, known as endotheliumderived relaxing factor (EDRF), which aided in the relaxation of blood vessels, and prompted further research into the endothelium's role as a potential regulator of vascular homeostasis.

In 1980, Furchgott demonstrated the obligatory role of the endothelium and EDRF in modulating vascular tone, and that acetylcholine (ACh), despite its very potent vasodilating action in vivo, does not always produce relaxation of isolated preparations of blood vessels in vitro (Furchgott and Zawadzki, 1980). The landmark study demonstrated that relaxation of isolated preparations of rabbit thoracic aorta and other blood vessels in vitro by ACh requires the presence of endothelial cells, and that ACh, acting on muscarinic receptors of these cells, stimulate the release of a substance that causes relaxation of the vascular smooth muscle. Furchgott and Zawadzki proposed that the response could potentially be one of the principal mechanisms of ACh-induced vasodilation 
in vivo, and concluded blood vessel dilation and constriction was regulated by endothelial cell activation. Over the next decade, EDRF was discovered to be synonymous with nitric oxide (Ignarro et al., 1987; Palmer et al., 1987; Günther et al., 1992).

\section{Endothelium Response to Shear Stress}

Many blood vessels within the human body respond to blood flow increases (i.e., shear stress) by dilating (Corretti et al., 2002). Shear stress is an applied force or pressure exerted against the surface and layers of the skin as tissues slide in opposite but parallel planes (TheFreeDictionary.com, 2011). Thus, when the body increases vascular blood flow, an increase in shear stress has been reported to stimulate the release of a principal vasodilatory mediator from the endothelium, called endothelium-derived nitric oxide (NO) (Corretti et al., 2002; Olesen et al., 1988). Nitric oxide has proved to be the regulatory factor between the endothelial cells and smooth muscle cells, acting as the chief endothelial vasodilator (alongside prostacyclin), and commanding the smooth muscle cells to relax and the vasculature to dilate.

Although the precise mechanism for the acute detection of shear forces and subsequent signal transduction to modulate vasomotor tone are not fully understood, it is known that the endothelial cell membrane contains calciumactivated potassium channels that open in response to shear stress (Cooke et 
al., 1991; Corretti et al., 2002; Miura et al., 2001; Olesen et al., 1988; van Breemen et al., 1997). The effect of potassium channel opening is to hyperpolarize the endothelial cell, increasing the driving force for cytoplasmic calcium entry (no voltage-gated calcium channels exist in endothelial cells). Calcium influx further activates an enzyme, endothelial nitric oxide synthase (eNOS), and the subsequent generation of NO (from L-arginine biosynthesis) appears to account for the dilation response (Joannides et al., 1995; Pohl et al., 1986). eNOS gene transcription can also remain activated over longer periods of time (many minutes or hours), resulting in continued NO generation (and dilation) if shear stress is maintained at high levels (Corretti et al., 2002). Additionally, NO production and shear stress maintenance also work to inhibit interaction between platelets, leukocytes, and the vessel wall, and inhibit smooth muscle migration and proliferation (Halcox \& Deanfield, 2004).

The calcium-sensitive NO release prompts diffusion to the underlying smooth muscle cells. Specifically, NO stimulates soluble guanylate cyclase, which prompts cyclic GMP activation of protein kinase G. The activation of protein kinase $\mathrm{G}$ stimulates phosphorylation of myosin light chain phosphatase, inactivation of myosin light-chain kinase (MLCK), and dephosphorylation of the myosin light chain. These phosphorylation events lead to a decrease in intracellular calcium (inhibit L-type calcium channels and stimulate sarcoplasmic reticulum calcium-pump ATPase, or reuptake of calcium into the intracellular 
stores), and further induces smooth muscle relaxation or vasodilation (Günther et al., 1992; Surks, 2007; van Breemen et al., 1997; Webb, 2003).

\section{Flow-Mediated, or Endothelium-Dependent Dilation}

Today, the most widely used and well-validated noninvasive technique for measuring peripheral endothelial function is ultrasound imaging during flowmediated dilation (FMD) of the brachial artery (Agewall et al., 2001; Corretti et al., 2002). Often referred to as EDD, endothelium response to FMD techniques provides a surrogate measure of endogenous NO bioavailability within the endothelium layer and can help identify the presence of endothelial dysfunction. Flow-mediated dilation quickly became the preferred means of providing a noninvasive index of endothelial health in both healthy and disease populations (Celermajer et al., 1992; Celermajer et al., 1993; Chowienczyk et al., 1992; Corretti et al., 1995; Corretti et al., 2002; Lieberman et al., 1996; Raitakari \& Celermajer, 2000; Roman et al., 2006; Williams et al., 1996) given its ability to suitably assess early preclinical stages of atherosclerosis or in instances of repeated measurement. Endothelium-dependent dilatation of the brachial arteries has appeared to correlate closely with that of coronary arteries (Anderson et al., 1995).

Studies report that whereas non-diseased arteries exhibited endotheliumdependent dilation in response to vasoactive stimuli, lack of dilatation has been 
demonstrated in diseased arteries, such as the effects from atherosclerosis (Celermajer et al., 1992; Ludmer et al., 1986; Lieberman et al., 1996). Additional studies have demonstrated that the presence of cardiovascular risk factors such as cigarette smoking, Type 2 diabetes mellitus, hypercholesterolemia, or increasing age (Adams et al., 1998; Anderson et al., 1995; Vogel et al., 1996; Williams et al., 1996) is also associated with abnormal endothelium-dependent vasodilation.

\section{Endothelium-Independent Dilation by Nitroglycerin Administration}

Celermajer et al. (1992) were among the first to study the effects of the FMD response of the brachial artery as an indicator of endothelium-dependent response to shear stress. The study also looked at endothelium-independent dilation (EID) response of the artery by measuring peak dilation after administration of NTG, an exogenous NO donor, and its facilitation of vascular smooth muscle relaxation. While the brachial artery responds to EDD generated reactive hyperemia via the endothelium's endogenous production of $\mathrm{NO}$ and its subsequent affect on smooth muscle, NTG helps ensure that abnormalities of FMD are due truly to endothelial dysfunction and the result of abnormal NObioavailability, and not necessarily smooth muscle insensitivity to nitric oxide (Bressler et al., 2000; Ducharme et al., 1999; Pepe et al., 2004; Roman et al., 2006). Specifically, NTG is converted to NO in the body by mitochondrial aldehyde dehydrogenase and does not require the endothelium to generate the 
potent vasodilator. Thus, smooth muscle relaxation will occur regardless of any endothelial dysfunction present.

Nitroglycerin-mediated dilation in adult populations is well documented among diseased populations. A study by Gokce et al. (2001) reported that both FMD $(p<0.001)$ and NTG-mediated dilation $(p<0.001)$ were significantly impaired in hypertensive versus normotensive subjects, and that race was a significant factor in vasodilatory response to NTG (black: $17.7 \pm 7.5$ vs. white: $15.0 \pm 6.2 \%$, $p=0.02)$. In another study, Dengel et al. (2008) reported greater percent EID and percent FMD among female adult survivors of acute lymphoblastic leukemia (ALL) compared to males after correcting for baseline brachial artery diameter. Furthermore, nitrate-mediated EID among those who received chemotherapy plus radiation or chemotherapy alone were not significantly different when compared to a healthy comparison group; yet the healthy comparison group elicited significantly greater FMD response than both the chemotherapy only and chemotherapy plus radiation groups (Dengel et al., 2008). Mizia-Stec et al. (2007) reported similar significance in relation to greater percent FMD in females compared to males $(p<0.05)$; however, similar results were not reported in EID. Additionally, a study by Doupis et al. (2011) reported that diabetes and obesity negatively significantly affect endothelial cell function in adults, while smooth muscle cell function was only affected by diabetes. The following research 
underscores the importance of assessing both FMD and EID in relation to vascular health.

To date, EID time course is more often performed among adult populations, and requires adequate time to accurately measure true dilation response given time courses often vary from person to person. A previous study in adults reported that a single dose $(0.4 \mathrm{mg})$ of NTG, sublingual or spray, stimulates a maximal brachial artery vasodilator response 3-min post-NTG administration during EID testing (Ducharme et al., 1999). In another adult study, Bressler et al. (2000) found that peak dilation occurred at approximately 5-min in coronary artery disease patients following a single dose $(0.3 \mathrm{mg})$ of NTG, with many individual peak responses occurring between 3- and 5-min. Finally, Thelen et al. (2008) reported peak EID response at 5-min post-NTG administration in a sample of healthy young adults, supporting the notion that reporting percent dilation at 3-min significantly underestimates true EID.

To our knowledge, no studies to date have described the time course of the EID response in children and adolescents. It is possible that the time course of peak EID in youth could be different than adults. Indeed, differences in EDD time course in youth have been described by Järvisalo et al. (2002), who observed that peak response occurs later in children and adolescents (79-sec) when compared to adults (Berry, Skyrme-Jones, \& Meredith, 2000). 


\section{CHAPTER 3. METHODOLOGY}


The study protocol was reviewed and approved by the University of Minnesota Institutional Review Board (IRB). Additionally, the procedures followed in the study adhered to the University of Minnesota's IRB and the Health Insurance Portability and Accountability Act (HIPAA) guidelines.

\section{Study Population}

One hundred ninety-eight healthy children and adolescents (113 males, 85 females) between the ages of 6 and 18 years (mean age $13.9 \pm 0.2 \mathrm{yrs}$ ) were assessed for EID up to 5-min. A smaller sub-group ( $\mathrm{N}=20 ; 12$ males, 8 females) was utilized to further compare gender differences in percent dilation up to 8-min. Subject data was previously collected within a University of Minnesota IRBapproved study of healthy siblings of cancer survivors, and another University of Minnesota IRB-approved study of adiposity among healthy children. All subjects submitted written informed assent and consent for participation in the study. Subjects were taken from community-based study protocols, and were otherwise considered healthy individuals. Prior to vascular testing, subjects were asked to fast for 12-hr prior to testing, as well as abstain from caffeine ingestion leading up to study commencement. Subjects were instructed not to take any of their usual morning medications until testing was complete. A study physician and/or certified nurse practitioner was present to review study procedures and evaluation plans, prescription medications, and conduct comprehensive medical examinations including current and past medical history, review of systems (with 
particular attention to cardiovascular and endocrine issues), family history (with particular attention to cardiovascular disease and diabetes), and a physical examination. A urine-based pregnancy test was completed for female participants when applicable.

\section{Measurements}

\section{Anthropometric and Blood Pressure Assessments}

Height and weight were obtained using a standard stadiometer (Ayrton, Model S100, Prior Lake, MN, USA) and electronic scale (ST Scale-Tronix, Serial No. 5002-8893, White Plains, NY, USA), respectively. Body Mass Index (BMI) was calculated as weight in kilograms divided by height in meters squared. Tanner "pubertal" stage was determined by trained pediatricians (Tanner, 1962). Body composition was obtained using dual energy X-ray absorptiometry (DXA) (Prodigy, Versions 10.5, 3M, Madison, WI, USA). Seated blood pressure was obtained on the control arm using an automatic sphygmomanometer (Colin Press-Mate, Model BP-8800C, San Antonio, TX, USA) after 5-min of quiet rest in the supine position.

\section{Vascular Assessment}

Testing was performed in the Vascular Biology Laboratory in the University of Minnesota Clinical and Translational Science Institute. All the vascular studies were performed in a quiet, temperature-controlled environment 
$\left(22^{\circ} \mathrm{C}-23^{\circ} \mathrm{C}\right)$. Endothelium-independent dilation was assessed using $0.3 \mathrm{mg}$ sublingual NTG, the dose considered appropriate for children by the University of Minnesota Institutional Review Board.

Following 15-min of quiet rest in the supine position, vascular images were obtained using a conventional ultrasound scanner (Acuson, Sequoia 512, Siemens Medical Solutions USA, Inc., Mountain View, CA, USA) with an $8.0 \mathrm{MHz}$ linear array probe held in place by a stereotactic arm. This system was interfaced with a standard personal computer equipped with a data acquisition card for attainment of radio frequency ultrasound signals from the scanner. All arterial images were triggered and captured at the $\mathrm{R}$ wave of the electrocardiogram (end-diastolic diameter), then digitized and stored on a personal computer for later off-line analysis using electronic wall-tracking software (Vascular Research Tools 5, Medical Imaging Application, LLC, lowa City, IA, USA).

Brachial artery diameter was assessed continuously for 5-min post-NTG administration. All image files were averaged over 10 -sec periods and peak dilation during the study was defined as the greatest percent change from resting baseline brachial artery diameter. Digital image analysis was performed by the same trained reader blinded to group assignments. 


\section{Statistical Analysis}

SPSS version 17.0 (SPSS, Chicago, IL, USA) was used for statistical analyses. Results are expressed as mean \pm standard error of the mean (SEM). An independent sample t-test was used to compare demographic characteristics by gender. Univariate analysis of co-variance (ANCOVA) with Bonferroni post hoc tests was used to compare percent dilations by genders while accounting for baseline diameter differences and Tanner "pubertal" stage, as well as age differences. A repeated measures analysis of variance with a Bonferroni post hoc test was also used to compare gender differences in percent dilation at different time points. Additionally, a chi-square test for trend was performed to assess differences in Tanner "pubertal" stage and race.

A univariate analysis of co-variance was additionally performed to assess differences both within and between genders and Tanner "pubertal" stages. Specifically, gender peak brachial artery dilation and time to peak dilation were stratified into Tanner "pubertal" stage categories (Stages I and II vs. Stages III, $\mathrm{IV}$, and V) to verify any existing differences that may exist within each gender. Similarly, Tanner Stages I and II were grouped together and compared to Tanner Stages III, IV, and V, relative to peak dilation and time to peak dilation to verify further differences that may exist between genders. 
An alpha value of 0.05 was used to signify statistical significance. Time to peak dilation was calculated by identifying the time point at which each individual reached maximal change in percent dilation, and then averaged among the subject population. 
CHAPTER 4. RESULTS 
Mean demographic data among the study population $(\mathrm{N}=198 ; 113$ males, 85 females) are presented in Table 1. A significant difference in height $(p=0.007)$, percent body fat $(p<0.0001)$, total fat mass $(p=0.011)$, and total lean mass $(p<0.0001)$ was reported between genders. Tanner "pubertal" stage was significantly different between males and females $(p=0.002)$, with the largest percentage residing within Stages IV and V. Furthermore, no significant difference was found within stratifications by gender and Tanner Stage categories (Table 2). Age was not significantly different between males and females $(13.8 \pm 0.2$ vs. $14.1 \pm 0.3 \mathrm{yrs}, p=0.4)$. Race was also observed to lack any significant differences between genders $(p=0.7)$.

Time to peak dilation after $0.3 \mathrm{mg}$ sublingual NTG administration was 4min, 28-sec (males: 4-min, 28-sec vs. females: 4-min, $26-\mathrm{sec}, p=0.6$ ), with a peak dilation of $24.9 \pm 0.4 \%$ (males: $24.8 \pm 0.5$ vs. females: $25.3 \pm 0.6 \%, p=0.6$ ) for subjects reporting data up to 5-min. Baseline brachial artery diameter was significantly greater in males than females, respectively $(3.5 \pm 0.0$ vs. $3.0 \pm 0.0$ $\mathrm{mm}, p<0.0001)$.

A significant $($ all $p<0.001)$ difference in peak brachial artery dilation response to NTG was reported at 3 vs. 4 -min, 4 vs. 5 -min, and 3 vs. 5 -min (Fig. 1). There was a significant $(p<0.05)$ difference in EID between males and 
females; however, significance was lost after accounting for baseline artery diameter $(24.7 \pm 0.5$ vs. $25.2 \pm 0.6 \%, p=0.6)$.

In the sub-group with EID data up to 8-min post-NTG administration $(\mathrm{N}=20 ; 12$ males, 8 females), the average time to peak dilation was 4-min, 24-sec (males: 4-min, 27-sec, females: 4-min, 19-sec), with an average change in peak dilation of $23.8 \pm 1.2 \%$ (male: $22.4 \pm 1.6 \%$, females: $25.8 \pm 1.8 \%$ ). A significant $(p<0.05)$ difference existed at 3 vs. 4 -min, 3 vs. 5 -min, 3 vs. 6 -min, 3 vs. 7 -min, and 3 vs. 8-min over an 8-min total time course (Fig. 2). Similar to the entire group, we observed a significant $(p<0.05)$ difference reported in EID between male and female genders in the small sub-group; however, significance disappeared after correcting for brachial artery diameter (males: $24.1 \pm 1.4 \%$ vs. females: $23.3 \pm 1.9 \%, p=0.7)$. 
CHAPTER 5. DISCUSSION 
The findings of the present study indicate that, among healthy children and adolescents, maximal brachial artery dilation following the administration of $0.3 \mathrm{mg} \mathrm{NTG}$ occurs at 4-min, $28-\mathrm{sec}$, with an average change in peak dilation of $24.9 \pm 0.4 \%$. EID at 5-min significantly differed from dilation response at both 3 min and 4-min time points, suggesting that reporting values prior to 5-min may underestimate the true EID response. These findings are similar to a previous report of EID in young adults (Thelen et al., 2008), which demonstrated EID response to NTG in a healthy adult population occurred approximately 5-min following post-NTG administration.

The smaller sub-group of 20 subjects, on which we had data up to 8 -min post-NTG, demonstrated an average time to peak dilation of 4-min, 24-sec, with a change in peak dilation of $23.8 \pm 1.2 \%$ (male: $22.4 \pm 1.6$ vs. females: $25.8 \pm$ $1.8 \%)$. The sub-group was analyzed to examine whether peak dilation occurred beyond 5-min. Specifically, the question remained following initial analysis of the entire study population whether 5-min provides ample time to capture true EID in children and adolescent. Of the individuals in the larger population, 20 of these subjects had data reported up to 8-min. However, we observed no further significant dilation beyond the 4-min time point. Thus, our results indicate that measuring brachial artery diameter beyond a 5-min time course may not be necessary in children and adolescent populations. Our data follow similar conclusions reported in subjects with clinical evidence of coronary artery disease 
(Bressler et al., 2000), in which peak dilations ranging between 3 and 5-min were reported. Although Thelen et al. (2008) and Bressler et al. (2000) found similar results as demonstrated within our research results; our study is novel in that we examined EID response in children and adolescents.

The present study also supports previous observations regarding the role of brachial artery diameter in peak dilation response (i.e., smaller baseline diameter generally equals higher peak dilation response) and its impact on vascular function. In general, females tend to have higher EID compared to males. The practical implication is that larger baseline diameter, as is commonly seen in men, results in reduced dilation responses compared to the relatively smaller arteries of women (Adams et al., 1996; Adams et al., 1998; Dengel et al., 2010; Juonala et al., 2008; Kapuku et al., 2004). We found a significant difference in peak EID between males and females; however, significance disappeared upon adjusting for differences in baseline brachial diameters, suggesting these previous observations in adults extend to children.

Furthermore, the reported difference supports our hypothesis that smaller resting brachial artery diameter in females results in larger dilation response compared to males. Similar responses to resting brachial artery diameter are reported in adult populations as well. These findings underscore the importance of adjusting for baseline brachial artery diameter when comparing response by gender in children and adolescents. 
Studies show that differences in EID and smooth muscle function between males and females may be related to hormonal differences between genders (Thompson et al., 2003). Within the present study, both peak brachial artery dilation and time to peak dilation were not significantly different between males and females following stratification into Tanner "pubertal" stage categories. Similarly, no significant difference was observed within gender following similar stratification. Furthermore, no significant hormonal differences both within and between genders were concluded. Research has demonstrated that both endothelial and smooth muscle cells in vascular beds have receptors for estrogen, progesterone, and testosterone, making the quantity of sex hormone receptors present in males and females an important consideration factor (Thompson et al., 2003). Females also have more estrogen receptors in their arteries than males following maturation, and may be more sensitive to vasodilators (Collins et al., 2005). Normal vascular function may be maintained in premenopausal women by the protective effect of estrogen, shown to balance lipids and facilitate nitric oxide production (Chambliss et al., 2002). Yet, the effect of estrogen on receptors in smooth muscle may be age-related, showing greater sensitivity in young, mature subjects as opposed to postmenopausal women (Sherwood et al., 2007). EID has shown no significant differences at different phases of the menstrual cycle (Williams et al., 2001; English et al., 1998). 
Strengths of the study include the relatively large sample size, balance between genders, and the uniform approach and analysis of the vascular data among the entire study population of 198 subjects. Potential limitations of the following study are that subjects were drawn from a relatively homogenous population, which may pose a potential problem in generalizing these findings to a heterogeneous population including less healthy subjects. Also, we used a smaller dose of NTG $(0.3 \mathrm{mg})$ than is commonly used in the adult population, so we cannot rule out the time course of dilation may differ at a dose of $0.4 \mathrm{mg}$. Past research has utilized cumulative nitrate-mediated dosing strategies (Järvisalo et al., 2002; Järvisalo et al., 2004); however, no studies to date have assessed response relative to per kilogram of weight. 
CHAPTER 6. CONCLUSION 
In this study population of healthy children and adolescents, maximal EID response was observed at approximately 4-min, 30-sec, post-NTG administration. Significant differences in EID were observed at 3 vs. 4-min, as well as 4 vs. 5 -min among the entire study population. Therefore, these data suggest that reporting EID at 3 or 4-min may underestimate the true EID peak in children and adolescents. Future research is needed to assess how cardiovascular disease risk factors in less healthy children and adolescent populations affect the time course of brachial artery dilation in response to NTG administration. 
CHAPTER 7. REFERENCES 
Adams M, Robinson J, Sorensen K, Deanfield J, Celermajer D. Normal ranges for brachial artery flow-mediated dilation: a non-invasive ultrasound test of arterial endothelial function. $J$ Vasc Invest 1996;2:146-150.

Adams M, Robinson J, McCredie R, Seale J, Sorensen K, Deanfield J, Celermajer D. Smooth muscle dysfunction occurs independently of impaired endothelium-dependent dilation in adults at risk of atherosclerosis. J Am Coll Cardiol 1998;32:123-127.

Agewall S, Doughty RN, Bagg W, Whalley GA, Braatvedt G, Sharpe N. Comparison of ultrasound assessment of flow-mediated dilatation in the radial and brachial artery with upper and forearm cuff positions. Clin Physiol 2001;21(1):9-14.

Anderson TJ, Uehata A, Gerhard MD, Meredith IT, Knab S, Delagrange D, Lieberman EH, Ganz P, Creager MA, Yeung AC, Selwyn S. Close relation of endothelial function in human coronary and peripheral circulations. $J$ Am Coll Cardiol 1995;26:1235-1241.

Berry KL, Skyrme-Jones RA, Meredith IT. Occlusion cuff position is an important determinant of the time course of magnitude of human brachial artery flow-mediated dilation. Clin Sci (Colch) 2000;99:261-267. 
Bressler B, Chan S, Mancini G. Temporal response of brachial artery dilation after occlusion and nitroglycerin. Am J Cardiol 2000;85(3):396-400.

Celermajer D, Sorensen K, Gooch V, Spielgelhalter D, Miller O, Sullivan I, Lloyd J, Deanfield J. Non-invasive detection of endothelial dysfunction in children and adults at risk of atherosclerosis. Lancet 1992;340:1111-1115.

Celermajer D, Sorensen K, Spiegelhalter D, Georgakopoulos D, Deanfield J. Aging is associated with endothelial dysfunction in healthy men years before the age-related decline in women (Abstract). Circulation 1993; 88:I78.

Chambliss K, Shaul P. Estrogen modulation of endothelial nitric oxide synthase. Endocr Rev 2002;23:665-686.

Chowienczyk P, Watts G, Cockcroft J, Ritter J. Impaired endothelium-dependent vasodilatation of forearm resistance vessels in hypercholesterolemia. Lancet 1992;340:1430-1432.

Cines DB, Pollak ES, Buck CA, Loscalzo J, Zimmerman GA, McEver RP, Pober JS, Wick TM, Konkle BA, Schwartz BS, Barnathan ES, McCrae KR, Hug 
BA, Schmidt AM, Stern DM. Endothelial cells in physiology and in the pathophysiology of vascular disorders. Blood 1998;91:3527-3561.

Collins P, Rosano GM, Sarrel PM, Ulrich L, Adamopoulos S, Beale CM, McNeill JG, Polle-Wilson PA. Coronary heart disease/myocardial infarction: 17 beta-estradiol attenuates acetylcholine-induced coronary arterial constriction in women but not men with coronary heart disease. Circulation 1995;92(1):24-30.

Cooke JP, Rossitch E, Andon NA, Loscalzo J, Dzau VJ. Flow activates an endothelial potassium channel to release an endogenous nitrovasodilator. J Clin Invest 1991;88:1663-1671.

Corretti M, Plotnick G, Vogel R. Technical aspects of evaluating brachial artery vasodilation using high-frequency ultrasound. Am J Physiol 1995; 268:H1397-H1404.

Corretti M, Anderson T, Benjamin E, Celermajer D, Charbonneau F, Creager M, Deanfield J, Drexler H, Gerhard-Herman M, Herrington D, Vallance P, Vita J, Vogel R. International Brachial Artery Reactivity Task Force. Guidelines for the ultrasound assessment of endothelial-dependent flow mediated vasodilation of the brachial artery. J Am Coll Cardiol 2002,39(2):257-265. 
Dengel D, Ness K, Glasser S, Williamson E, Baker K, Gurney J. Endothelial function in young adult survivors of childhood acute lymphoblastic leukemia. J Pediatr Hematol Oncol 2008;30(1):20-25.

Dengel D, Jacobs D, Steinberger J, Moran A, Sinaiko A. Gender differences in vascular function and insulin sensitivity in young adults. Clin Sci 2010; 120(4):153-160.

Doupis J, Rahangdale S, Gnardellis C, Pena SE, Malhotra A, Veves A. Effects of diabetes and obesity on vascular reactivity, inflammatory cytokines, and growth factors. Obesity 2011;19(4):729-735.

Ducharme A, Dupuis J, McNicoll S, Harel F, Tardif J. Comparison of nitroglycerin lingual spray and sublingual tablet on time of onset and duration of brachial artery vasodilation in normal subjects. Am J Cardiol 1999; 84(8):952-954.

English JL, Jacobs JO, Green G, Andrews TC. Effect of the menstrual cycle on endothelium-dependent vasodilation of the brachial artery in normal young women. Am J Cardiol 1998;82:256-258. 
Furchgott RF, Zawadzki JV. The obligatory role of endothelial cells in the relaxation of arterial smoot muscle by acetylcholine. Nature 1980;288(5789):373-376 .

Günther J, Dhein S, Rösen R, Klaus W, Fricke U. Nitric oxide (EDRF) enhances the vasorelaxing effect of nitrendipine in various isolated arteries. Basic Res Cardiol 1992;87:452-460.

Gokce N, Holbrook M, Duffy SJ, Demissie S, Cupples LA, Biegelsen E, Keaney JF, Loscalzo J, Vita JA. Effects of race and hypertension on flow-mediated and nitroglycerin-mediated dilation of the brachial artery. Hypertension $2001 ; 38(6): 1349-1354$.

Halcox JPJ, Deanfield JE. Endothelial cell function testing: how does the method help us in evaluating vascular status? Acta Paediatr Suppl 2004;446:4854.

Ignarro L, Byrus R, Buga G, Wood K. Endothelium-derived relaxing factor from pulmonary artery and vein possesses pharmacologic and chemical properties identical to those of nitric oxide radical. Circ Res 1987;61:866879. 
Järvisalo MJ, Rönnemaa T, Volanen I, Kaitosaari T, Kallio K, Hartiala JJ, Irjala K, Viikari JS, Simell O, Raitakari O. Brachial artery dilatation responses in healthy children and adolescents. Am J Physiol Heart Circ Physiol 2002; 282(1):H87-92.

Järvisalo MJ, Lehtimäki T, Raitakari O. Determinants of arterial nitrate-mediated dilatation in children: role of oxidized low-density lipoprotein, endothelial function, and carotid intima-media thickness. Circulation 2004;109:28852889.

Joannides R, Haefeli WE, Linder L, et al. Nitric oxide is responsible for flowdependent dilatation of human peripheral conduit arteries in vivo. Circulation 1995;91:1314-1319.

Juonala M, Kähönen M, Laitinen T, Hutri-Kähönen N, Jokinen E, Taittonen L, Pietikäinen M, Helenius H, Viikari J, Raitakari O. Effect of age and sex on carotid intima-media thickness, elasticity and brachial endothelial function in healthy adults: The Cardiovascular Risk in Young Finns Study. Eur Heart J 2008;29:1198-1206. 
Kapuku G, Treiber F, Hartley B, Ludwig D. Gender influences endothelialdependent arterial dilation via arterial size in youth. Am J Med Sci 2004; 327(6):305-309.

Lieberman EH, Gerhard MD, Uehata A, Selwyn AP, Ganz P, Yeung AC, Creager MA. Flow-induced vasodilation of the human brachial artery is impaired in patients $<40$ years of age with coronary artery disease. Am J Cardiol 1996;78:1210-1214.

Ludmer PL, Selwyn AP, Shook TL, Wayne RR, Mudge GH, Alexander RW, Ganz P. Paradoxical vasoconstriction induced by acetylcholine in atherosclerotic coronary arteries. N Engl J Med 1986;315(17):1046-1051.

Miura $\mathrm{H}$, Wachtel RE, Liu Y, et al. Flow-induced dilation of human coronary arterioles: important roe of $\mathrm{Ca}(2+)$-activated $\mathrm{K}(+)$ channels. Circulation 2001;103:1992-1998.

Mizia-Stec K, Gasior Z, Mizia M, Haberka M, Holecki M, Zwolinska W, Kanczuga $\mathrm{K}$, Skowerski M. Flow-mediated dilation and gender in patients with coronary artery disease: arterial size influences gender differences in flowmediated dilation. Echocardiography 2007;24(10):1051-1057. 
Olesen SP, Clapham DE, Davies PF. Haemodynamic shear stress activates a K+ current in endothelial cells. Nature 1988;331:168-170.

Palmer R, Ferridge A, Moncada S. Nitric oxide release accounts for the biological activity of endothelium-derived relaxing factor. Nature 1987;327:524-526.

Pepe A, Lombardi M, Takacs I, Positano V, Panzarella G, Picano E. Nitrateinduced coronary vasodilation by stress-magnetic resonance imaging: a novel noninvasive test of coronary vasomotion. J Magn Reson Imaging 2004;20:390-394.

Pohl U, Holtz J, Busse R, Bassenge E. Crucial role of endothelium in the vasodilator response to increased flow in vivo. Hypertension 1986;8:3744.

Raitakari O, Celermajer D. Flow-mediated dilatation. Brit J Clin Pharmacol 2000;50:397-404.

Roman M, Naqvi T, Gardin J, Gerhard-Herman M, Jaff M, Mohler E. Clinical application of noninvasive ultrasound in cardiovascular risk stratisfication: A report from the American Society of Echocardiography and the Society 
of Vascular Medicine and Biology. J Am Soc Echocardiogr 2006;19:943954.

shear stress. 2011. In TheFreeDictionary.com. Farflex, Inc. Retrieved May 17, 2011, from http://medical-dictionary.thefreedictionary.com/shear+stress

Sherwood A, Bower JK, McFetridge-Durdle J, Blumenthal JA, Newby LK, Hinderliter AL. Age moderates the short-term effects of transdermal 17ßestradiol on endothelium-dependent vascular function in postmenopausal women. Arterioscler Thromb Vasc Biol 2007;27:1782-1787.

Surks HK. cGMP-dependent protein kinase I and smooth muscle relaxation: a tale of two isoforms. Circulation Research 2007;101(11):1078-1080.

Tanner JM. Growth at adolescence (2nd ed.). Oxford: Blackwell 1962;69-87.

Thelen A, Kelly A, Williamson E, Dengel D. Examining the time course of endothelium-independent dilation by nitroglycerin. Ultrasound Med Biol 2008;34(8):1217-1220.

Thompson J, Khalil RA. Gender differences in the regulation of vascular tone. Clin Exper Pharmacol Physiol 2003;30:1-15. 
van Breemen C, Skarsgard P, Laher I, McManus B, Wang X. Endotheliumsmooth muscle interaction in blood vessels. Clin Exper Pharmacol Physiol 1997;24:989-992.

Vogel RA, Coretti MC, Plotnick GD. Changes in flow-mediated brachial artery vasoactivity with lowering of desirable cholesterol levels in healthy middleaged men. Am J Card 1996;77:37-40.

Webb RC. Smooth muscle contraction and relaxation. Advan in Physiol Edu 2003;27:201-206.

Williams MRI, Westerman RA, Kingwell BA, Paige J, Blombery PA, Sudhir K, Komesaroff PA. Variations in endothelial function and arterial compliance during the menstrual cycle. J Clin Endocrinol Metab 2001;86:5389-5395.

Williams SB, Cusco JA, Roddy MA, ETC. Impaired nitric oxide in the local regulation of pulmonary vascular resistance in humans. J Am Coll Card 1996;27:567-574. 
Yoshida T, Kawano H, Miyamoto S, Motoyama T, Fukushima H, Hirai N, Ogawa $\mathrm{H}$. Prognostic value of flow-mediated dilation of the brachial artery in patients with cardiovascular disease. Intern Med 2006;45(9):575-579. 
Table 1. Mean ( \pm Standard Error of the Mean) Demographic Characteristics

\begin{tabular}{|c|c|c|c|c|}
\hline & $\begin{array}{c}\mathrm{ALL} \\
(\mathrm{N}=198)\end{array}$ & $\begin{array}{c}\text { MALE } \\
(n=113)\end{array}$ & $\begin{array}{c}\text { FEMALE } \\
(\mathrm{n}=85)\end{array}$ & $p$-value \\
\hline Age (yrs) & $13.9 \pm 0.2$ & $13.8 \pm 0.2$ & $14.1 \pm 0.3$ & 0.394 \\
\hline Race & & & & 0.665 \\
\hline White & $152(77 \%)$ & $84(74 \%)$ & $68(80 \%)$ & \\
\hline Black & $30(15 \%)$ & 22 (19\%) & $8(9 \%)$ & \\
\hline American Indian & $4(2 \%)$ & $2(2 \%)$ & $2(2 \%)$ & \\
\hline Asian/Pacific Islander & $2(1 \%)$ & $1(1 \%)$ & $1(1 \%)$ & \\
\hline Other/More than One Race & $10(5 \%)$ & $4(4 \%)$ & $6(8 \%)$ & \\
\hline Height (cm) & $163.1 \pm 0.9$ & $165.3 \pm 1.4$ & $160.2 \pm 1.1$ & 0.007 \\
\hline Weight (kg) & $61.7 \pm 1.4$ & $63.0 \pm 2.1$ & $60.0 \pm 1.7$ & 0.297 \\
\hline $\mathrm{BMI}\left(\mathrm{kg} / \mathrm{m}^{2}\right)$ & $23.1 \pm 0.4$ & $23.0 \pm 0.5$ & $23.2 \pm 0.5$ & 0.761 \\
\hline Percent Fat (\%) & $26.8 \pm 0.8$ & $23.4 \pm 1.1$ & $32.0 \pm 1.0$ & $<0.0001$ \\
\hline Total Fat Mass $(\mathrm{kg})$ & $17.5 \pm 0.9$ & $15.7 \pm 1.2$ & $20.1 \pm 1.1$ & 0.011 \\
\hline Total Lean Mass (kg) & $44.3 \pm 0.9$ & $47.3 \pm 1.4$ & $40.2 \pm 0.8$ & $<0.0001$ \\
\hline Baseline Diam. (mm) & $3.3 \pm 0.0$ & $3.5 \pm 0.0$ & $3.0 \pm 0.0$ & $<0.0001$ \\
\hline Tanner Stage & & & & 0.002 \\
\hline Stage I & $14(8 \%)$ & $13(13 \%)$ & $1(1 \%)$ & \\
\hline Stage II & $18(10 \%)$ & $13(13 \%)$ & $5(6 \%)$ & \\
\hline Stage III & $16(9 \%)$ & $10(10 \%)$ & $6(8 \%)$ & \\
\hline Stage IV & $45(25 \%)$ & $21(20 \%)$ & $24(31 \%)$ & \\
\hline Stage V & $87(48 \%)$ & $45(44 \%)$ & $42(54 \%)$ & \\
\hline
\end{tabular}

BMI (body mass index); Baseline Diam. (baseline diameter);

$p$-values $<0.05$ demonstrate significant differences between genders. 
Table 2. P-values Among Gender and Tanner Stage Stratifications $p$-value

\section{Peak Brachial Artery Dilation (\%)}

Male: Tanner Stages I and II vs. III, IV, V

$p=0.532$

Female: Tanner Stages I and II vs. III, IV, V

$p=0.345$

Tanner Stages I and II: Male vs. Female

$p=0.827$

Tanner Stages III, IV, V: Male vs. Female

$p=0.651$

Time to Peak Dilation (secs)

Male: Tanner Stages I and II vs. III, IV, V

$p=0.496$

Female: Tanner Stages I and II vs. III, IV, V

$p=0.510$

Tanner Stages I and II: Male vs. Female

$p=0.386$

Tanner Stages III, IV, V: Male vs. Female

$p=0.972$ 


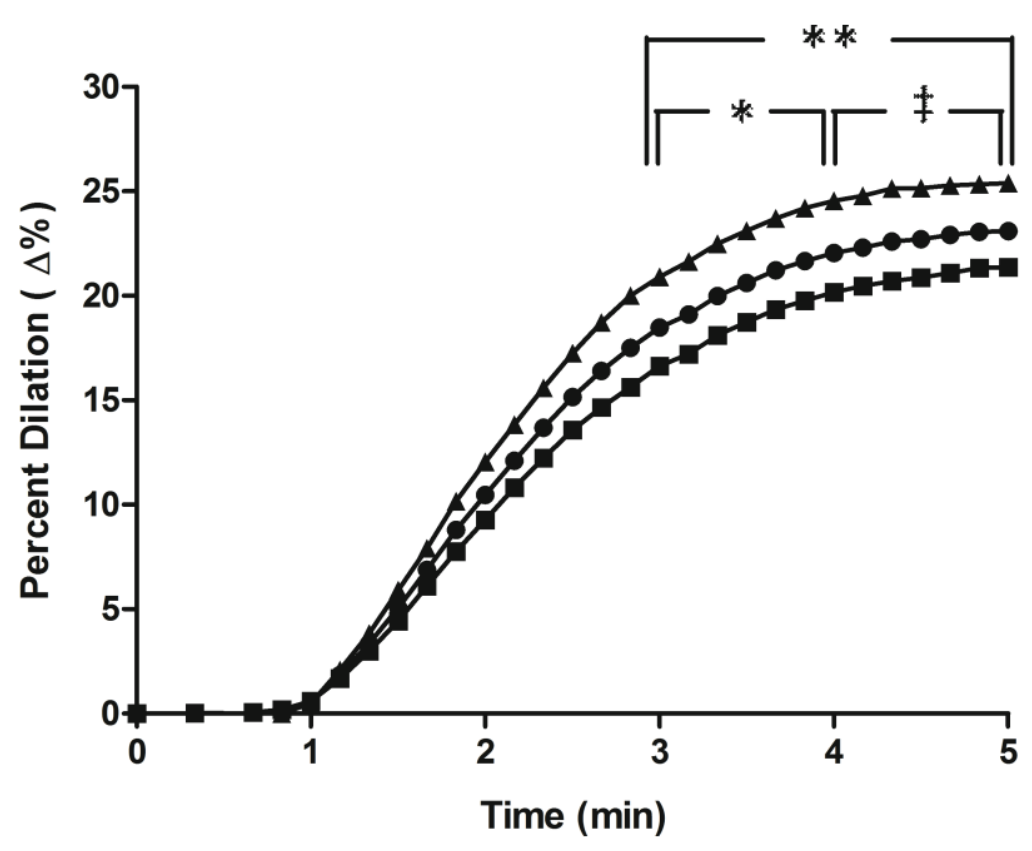

Figure 1. Endothelium-Independent Dilation Time Course Over 5 Minutes. Average time course of endothelial-independent dilation (EID) for the entire study population $(\mathrm{N}=198)(\bullet)$, as well as separated between males $(\boldsymbol{\square})$ and females

$(\boldsymbol{\Delta})$ from 0 to 5 -min. Significant $(p<0.05)$ differences were reported at 3 vs. 4 -min $\left({ }^{\star}\right), 3$ vs. 5 -min $\left(^{* \star}\right)$, and 4 vs. 5 -min $(\ddagger)$ within the entire study population; however, significance was lost after accounting for baseline artery diameter (24.7 \pm 0.5 vs. $25.2 \pm 0.6 \%, p=0.6)$. 


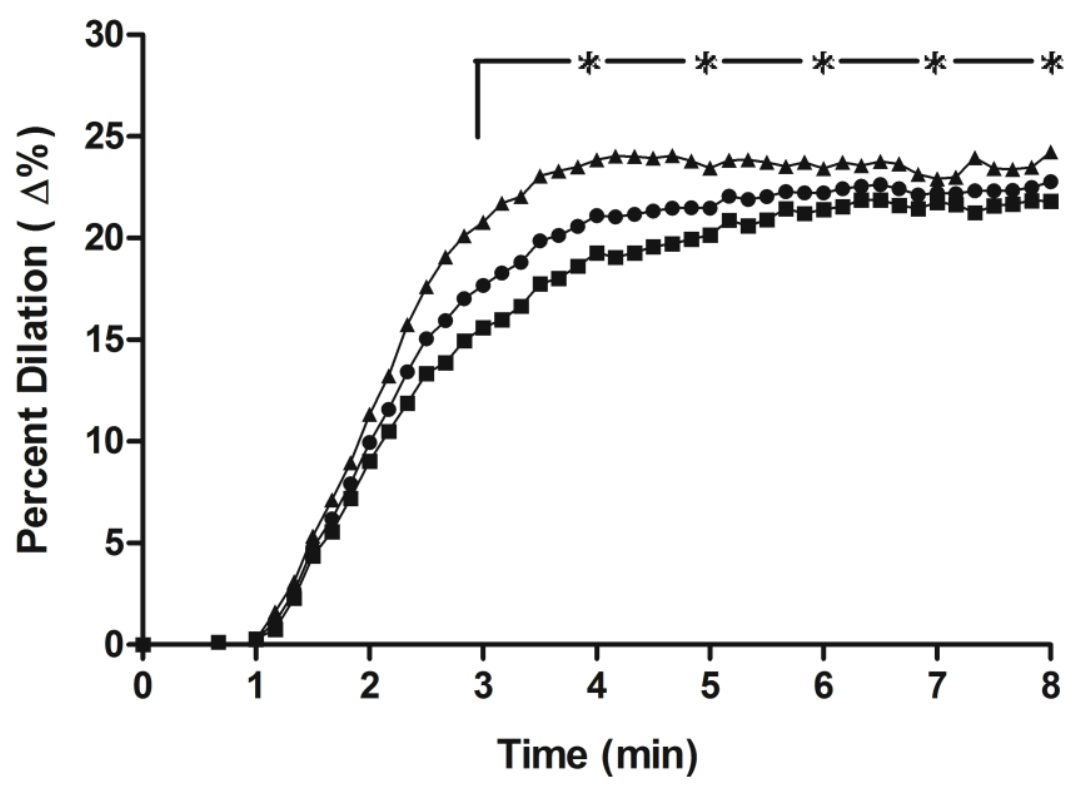

Figure 2. Endothelium-Independent Dilation Time Course Over 8 Minutes. Average time course of endothelial-independent dilation (EID) for the smaller sub-group population $(\mathrm{N}=20)(\bullet)$, as well as separated between males $(\boldsymbol{\bullet})$ and females $(\boldsymbol{\Delta})$ from 0 to 8 -min. Significant $(p<0.05)$ differences were reported at 3 vs. $4-\mathrm{min}, 3$ vs. 5 -min, 3 vs. $6-\mathrm{min}, 3$ vs. $7-\mathrm{min}$, and 3 vs. 8 -min (denoted *) within the population. A significant $(p<0.05)$ difference reported in EID between male and female genders in the small sub-group; however, significance disappeared after correcting for brachial artery diameter (males: $24.1 \pm 1.4 \%$ vs. females: $23.3 \pm 1.9 \%, p=0.7)$. 